\title{
The Seed Semipermeable Layer and Its Relation to Seed Quality Assessment in Four Grass Species
}

\author{
Yan Y. Lv't, Xue Q. He ${ }^{2 t}$, Xiao W. Hu ${ }^{1}$ and Yan R. Wang ${ }^{1 *}$ \\ ${ }^{1}$ The State Key Laboratory of Grassland Agro-ecosystems, College of Pastoral Agriculture Science and Technology, \\ Lanzhou University, Lanzhou, China, ${ }^{2}$ College of Animal Science and Technology, Northwest A\&F University, Yangling, China
}

OPEN ACCESS

Edited by:

Alma Balestrazzi,

University of Pavia, Italy

Reviewed by:

Khawar Jabran,

Duzce University, Turkey

Paola Leonetti,

Consiglio Nazionale delle Ricerche

(CNR), Italy

*Correspondence:

Yan R. Wang

yrwang@|zu.edu.cn

tThese authors are co-first authors and have contributed equally to this work.

Specialty section:

This article was submitted to Crop Science and Horticulture, a section of the journal

Frontiers in Plant Science

Received: 30 March 2017

Accepted: 19 June 2017

Published: 04 July 2017

Citation:

LV YY, He XQ, Hu XW and Wang YR (2017) The Seed Semipermeable Layer and Its Relation to Seed Quality Assessment in Four Grass Species.

Front. Plant Sci. 8:1175.

doi: 10.3389/fpls.2017.01175
The existence of a semipermeable layer in grass seeds has been extensively reported, yet knowledge of its influence on tests for seed viability and vigor that depend upon measurement of electrical conductivity (EC) is limited. This study determined the presence and location of the semipermeable layer, and its relation to seed viability and vigor assessment, in seeds of four important grass species-Elymus nutans Griseb., Lolium perenne L., Leymus chinensis (Trin.) Tzvel., and Avena sativa L. Intact seeds of E. nutans, Lolium perenne, and Leymus chinensis exhibited little staining with triphenyl tetrazolium chloride (TTC), and there were no differences in EC between seeds with different germination percentage (GP) $(P>0.05)$. After piercing the seed coat, however, all three species displayed positive staining with $\Pi T C$, along with a significant negative correlation between EC and GP (E. nutans: $R^{2}=0.7708$; Lolium perenne: $R^{2}=0.8414$; Leymus chinensis: $\left.R^{2}=0.859 ; P<0.01\right)$. In contrast, both intact and pierced seeds of $A$. sativa possessed a permeable seed coat that showed positive staining with TTC and EC values that were significantly negatively correlated with GP $\left[R^{2}=0.9071\right.$ (intact) and 0.9597 (pierced); $P<0.01$ ]. In commercial seed lots of $A$. sativa, a field emergence test indicated that EC showed a significant negative correlation with field emergence at two sowing dates $\left(R^{2}=0.6069, P<0.01\right.$ and $\left.0.5316, P<0.05\right)$. Analysis of seed coat permeability revealed the presence of a semipermeable layer located in the seed coat adjacent to the endosperm in E. nutans, Lolium perenne, and Leymus chinensis; however, no semipermeable layer was observed in $A$. sativa. This is the first report of the absence of a semipermeable layer in a grass species. The existence of a semipermeable layer is one of the most important factors affecting seed viability and vigor testing (based on EC measurement) in E. nutans, Lolium perenne, and Leymus chinensis. Increasing the permeability of the semipermeable layer, e.g., by piercing the seed coat, may permit the use of EC measurement to assess seed vigor in species that possess such a layer.

Keywords: electrical conductivity, grass species, permeability, seed vigor, semipermeable layer

\section{INTRODUCTION}

There are many traits that contribute to seed quality assessment, and seed vigor is the sum total of those properties of the seed that determine its potential activity and performance during germination and seedling emergence (Hampton and TeKrony, 1995). Seed vigor contributes directly to the economic success of commercial crops (Finch-Savage, 1995) and 
hence seed companies require methods of obtaining a reliable estimate of vigor in order to be able to supply growers with seed lots that possess high vigor. However, seed vigor remains difficult to assess, because it is a complex characteristic that is determined by a number of different factors.

Electrical conductivity (EC) is recommended as a convenient and validated method for assessing seed vigor in Pisum sativum L. (Matthews and Bradnock, 1967; Hampton and TeKrony, 1995), and it is widely used for vigor testing of large-seeded legumes, some small-seeded legumes, and Brassica pekinensis Rupr. (Hampton and TeKrony, 1995; Wang et al., 2004; Matthews et al., 2009; ISTA, 2016). However, in most grass species, with the exception of Festuca arundinacea Keng. (Han et al., 1995), EC values do not change with seed deterioration; examples are Bromus japonicus Thunb. ex Murr. (Ching and Schoolcraft, 1968), Lolium perenne L. (Hall and Wiesner, 1990), and Sorghum sudanense (Piper) Stapf. (Wang, 2003). Previous studies suggested that this phenomenon may be related to the high starch content in the embryo (Yi et al., 1994), the high content of insoluble substances (Han and Mao, 2000), or low EC values of grass seeds due to seed coat semipermeability (Wang, 2003).

A semipermeable layer, defined as a tissue that is permeable to water and gases but that restricts or impedes the exchange of some solutes (Beresniewicz et al., 1995a), exists widely in the seed coats of many species, such as Allium tuberosum Rottl. ex Spreng., Allium cepa L., Lycopersicon esculentum Mill., and Capsicum annuum L. (Beresniewicz et al., 1995a), × Triticosecale Wittm. ex A. Camus., Festuca sinensis Keng. and Bromus inermis L. (Zhou et al., 2013a), Cucumis sativus L. (Salanenka and Taylor, 2008; Salanenka et al., 2009), Lactuca sativa L. (Hill and Taylor, 1989), and Sorghum sudanense (Piper) Stapf.(Yan and Wang, 2008). The presence of a semipermeable layer may limit the exchange of substances between the seed and its external environment, and thus reduce the validity of methods for estimating seed performance based on seed coat permeability. For example, in Allium tuberosum, Allium cepa, Lycopersicon esculentum, and Capsicum annuum (Beresniewicz et al., 1995a), and in Sorghum sudanense (Wang, 2003), the presence of a semipermeable layer results in failure to stain with triphenyl tetrazolium chloride (TTC) unless the layer is punctured. Moreover, the presence of a semipermeable layer has been found to restrict electrolyte leakage in Sorghum sudanense (Wang, 2003) and Cucumis melo L. (Welbaum and Bradford, 1990), and leakage of amino acids in Allium tuberosum, Allium cepa, Lycopersicon esculentum, and Capsicum annuum (Taylor et al., 1995). In contrast, Brassica oleracea L., which lack a semipermeable layer, showed strong TTC staining and high rates of amino acids leakage in lower vigor seeds (Beresniewicz et al., 1995a; Taylor et al., 1995). These results imply that the presence of a semipermeable layer could affect TTC staining and EC testing, in the latter case by restricting solute leakage. However, although the presence (Hill and Taylor, 1989; Beresniewicz et al., 1995b; Salanenka and Taylor, 2008; Salanenka et al., 2009; Zhou et al., 2013a), location (Beresniewicz et al., 1995b; Yan and Wang, 2008; Zhou et al., 2013a), chemical composition (Beresniewicz et al., 1995a; Zhou et al., 2013a), and formation (Zhou et al., 2013b) of the semipermeable layer has been reported in several species, and been shown to vary between species, its importance in seed quality assessment has rarely been addressed.

Thus, we hypothesized that the presence of a semipermeable layer in grass species may be one of the principal factors that affect TTC staining and EC measurement in the assessment of grass seed quality. In this study, we aimed to determine (1) the presence and location of the semipermeable layer in seeds of four important forage grass species: Elymus nutans Griseb., Lolium perenne L., Leymus chinensis (Trin.) Tzvel., and Avena sativa L.; (2) how the semipermeable layer affects staining with TTC and the rate of solute leakage measured by an EC test, thus affecting vigor testing, and (3) whether EC testing can be used to predict seedling emergence when the species concerned does not possess a semipermeable layer.

\section{MATERIALS AND METHODS}

\section{Seeds and Seed Lots}

The presence and location of the semipermeable layer and seed coat permeability were determined using one seed lot for each species: E. nutans and A. sativa were harvested in 2006 from Gannan and Jingtai in Gansu Province, Lolium perenne and Leymus chinensis were obtained in 2007 from Inner Mongolia, China, and Oregon, United States, respectively. The primary viability for these seed samples was $>90 \%$.

A mixture of living and dead seeds was obtained by subjecting seeds to controlled deterioration as described by Hampton and TeKrony (1995). Germination percentage (GP) was $28-96 \%$ in E. nutans, 0-98\% in Lolium perenne, 12-94\% in Leymus chinensis, and $0-95 \%$ in A. sativa.

An additional 15 commercial seed lots of both E. nutans and A. sativa were used for field emergence tests on two sowing dates. Each of the seed lots had been harvested between 2011 and 2013 from different regions of Gansu Province, China, and the lots were provided by the Herbage Seed Testing Center of Lanzhou, China. The reported germination of the seed lots ranged from 70 to $82 \%$ for E. nutans and from 80 to $93 \%$ for A. sativa. All seed samples were stored dry at $4^{\circ} \mathrm{C}$ until used.

\section{Germination Test}

Germination percentage was determined in non-treated seed lots and in those subjected to the deterioration treatment. Three replicate groups of 50 seeds were sown in $8-\mathrm{cm}$ Petri dishes on two layers of filter paper moistened with distilled water. Water was added to the dishes as necessary to keep the filter paper moist during the test period. At the end of the germination test, the number of normal seedlings was counted.

\section{Imbibition and TTC Staining Test of Seeds}

Imbibition testing was carried out using three replicates of 100 seeds. Seeds were weighed, soaked in $100 \mathrm{~mL}$ of deionized water, and then incubated at $25^{\circ} \mathrm{C}$ for $24 \mathrm{~h}$. They were then surface-dried and weighed. The imbibition rate was estimated 
as imbibition $(\%)=$ (seed weight after imbibition/initial seed weight) $\times 100$ (Wang et al., 2002).

For TTC staining, intact seeds were soaked in water in a Petri dish for $1 \mathrm{~h}$. They were then stained with $1 \%(\mathrm{w} / \mathrm{v})$ TTC solution and kept in the dark at $20^{\circ} \mathrm{C}$ for $36 \mathrm{~h}$. Evaluation of the intensity of staining was performed according to International Seed Testing Association (Hampton and TeKrony, 1995) rules, and the results were divided into three categories: strong staining (seeds completely and well stained), slight staining (partially or completely stained, but faint), and no staining.

Punctured seeds were prepared by piercing the seed coat with a needle to overcome the physical barrier (Hill and Taylor, 1989) and then test imbibition rate and TTC staining was carried out as described above.

\section{EC Testing}

Electrical conductivity testing was performed using a conductivity meter (DST-A, AIP, Tianjin, China) on three replicates of 50 seeds for each sample. Each sample showed a different GP. Seeds were weighed, transferred to $100 \mathrm{~mL}$ of distilled water, stirred, covered, and incubated at $20^{\circ} \mathrm{C}$ for $24 \mathrm{~h}$. Distilled water was used as the control. The EC value ( $\mu \mathrm{s} \mathrm{cm}^{-1} \mathrm{~g}^{-1}$ ) of the incubation solution was measured after $24 \mathrm{~h}$, as described by Hampton and TeKrony (1995). The EC was calculated using the following equation:

$$
E C_{\mathrm{t}}=\left(E C_{\mathrm{s}}-E C_{\mathrm{c}}\right) / M_{\mathrm{s}}
$$

where $E C_{\mathrm{t}}$ is the relative $\mathrm{EC}, E C_{\mathrm{s}}$ denotes the $\mathrm{EC}$ of the sample, $E C_{\mathrm{c}}$ denotes the $\mathrm{EC}$ of the control, and $M_{\mathrm{S}}$ is the total mass of the sample.

Punctured seeds with a range of GPs were prepared as previously described (section "Imbibition and TTC Staining Test of Seeds"), and the EC values were determined as indicated above.

\section{Field Emergence Test of E. nutans and A. sativa Commercial Seed Lots}

A field emergence test of 15 seed lots was conducted in Xiahe County, in Gansu Province. Seeds were sown in soil by hand, $2-3 \mathrm{~cm}$ deep, in a nursery, on two different sowing dates (late April and late May). Each sample consisted of 100 seeds, with four replicates. The seedlings were evaluated after they had emerged from the soil and had grown to $2 \mathrm{~cm}$ in height; evaluation continued until no new seedlings emerged.

\section{Microscopic Analysis of Seed Coat and Endosperm}

Fifteen seeds of each species were randomly selected, and the lemma and palea were removed. The seeds were then placed at $20^{\circ} \mathrm{C}$ in an incubator for $24 \mathrm{~h}$, after which approximately $1 \mathrm{~mm}^{3}$ of the embryo surface attached to a small piece of endosperm tissue was removed using a razor blade. The tissue was prepared for semi-thin sections as described by Zheng and $\mathrm{Gu}$ (1993). In brief, the embryo tissue was soaked in 3\% (w/v) glutaraldehyde solution, and then pre-fixed for $6 \mathrm{~h}$ at $4^{\circ} \mathrm{C}$ before being rinsed three times for $10 \mathrm{~min}$ with phosphate buffer solution (PBS, 0.1 M, pH 7.2). The tissue was then fixed overnight in $1 \%(\mathrm{w} / \mathrm{v})$ osmic acid at $4^{\circ} \mathrm{C}$ and rinsed three times for $10 \mathrm{~min}$ with PBS. The seeds were next dehydrated using an ethanol series $(70,80,90$, and $100 \%$ ethanol successively for $15 \mathrm{~min}$ each). Ethanol dehydration was repeated twice and was followed by dehydration with epoxypropane. The tissue was then embedded with epoxypropane and Epon812 [1:1 ratio $(\mathrm{v} / \mathrm{v})]$ for polymerization $\left(35,45\right.$, and $60^{\circ} \mathrm{C}$ successively for $24 \mathrm{~h}$ each). The tissue was removed from the embedding mold and processed into a block using an ultramicrotome (LKD-2088 Ultratom V., Bromma, Sweden), and semi-thin sections $(2 \mu \mathrm{m})$ were cut. Finally, the sections were stained with $0.05 \%(\mathrm{w} / \mathrm{v})$ toluidine blue, sealed with Canada gum, and observed under a light microscope.

\section{Transmission Electron Microscopy (TEM) Analysis of the Semipermeable Layer}

The degree of lanthanum infiltration can be used to analyze the location of the semipermeable layer in the seed. Fifteen seeds were randomly selected for use in the TEM analysis. First, the lemma and palea were removed and the seeds were incubated at $20^{\circ} \mathrm{C}$. Then, individual seeds were soaked for $24 \mathrm{~h}$ in deionized water (seeds with a broken seed coat were discarded) and incubated in a $4 \%(\mathrm{w} / \mathrm{v})$ solution of the tracer compound lanthanum nitrate at $20^{\circ} \mathrm{C}$ for $24 \mathrm{~h}$. Part of the embryo surface was removed and cut into $1-\mathrm{mm}^{3}$ slices using a razor blade. An embedding block (containing the seed coat together with part of the endosperm) was prepared, and 70nm-thick sections were cut using an LKD-2088 ultramicrotome. The sections were stained with uranyl acetate for $1.5 \mathrm{~h}$, followed by citrate staining for 7-10 $\mathrm{min}$, and were then subjected to TEM imaging (JEM 1230 - JEOL, Tokyo, Japan). The position of the semipermeable layer was determined by the location of the lanthanum signal.

\section{Observation of the Semipermeable Layer by Energy-Dispersive X-ray Spectroscopy (EDX)}

A sufficient number of seeds, usually 15 seeds, with the lemma and palea removed, were soaked in deionized water at $20^{\circ} \mathrm{C}$ for $24 \mathrm{~h}$. Individual seeds were then soaked in $4 \%(\mathrm{w} / \mathrm{v})$ lanthanum nitrate solution at $20^{\circ} \mathrm{C}$ for $24 \mathrm{~h}$ and dried at room temperature. Sections approximately $100-\mathrm{nm}$ thick were then prepared as described previously for TEM. They were then examined using an EDX spectrometer (EDX; United States; KEVE2) and a scanning electron microscope (JSM5600LV; JEOL). The lanthanum spectrum was used to locate the position of the semipermeable layer.

\section{Statistical Analysis}

Linear regression analysis was used to represent the relationship between EC and GP/field emergence, using SPSS 19.0 (IBM Corp., Armonk, NY, United States). 


\section{RESULTS}

\section{Imbibition and TTC Staining}

In all four species, both intact and pierced seeds readily imbibed water, but the amount imbibed by pierced seeds was greater than that imbibed by intact seeds (Table 1). This indicated that the seed coat does not prevent water uptake. Further, non-aged seeds (intact seeds) germinated normally, indicating that gas exchange is not impaired by the seed coat. The TTC staining test, to evaluate uptake of vital stain into non-aged intact seeds, showed little strong TTC staining in E. nutans, Lolium perenne, and Leymus chinensis embryos; however, if the seed coats had been pierced, most of the embryos were strongly stained $(94,84$, and $89 \%$, respectively). This indicates that in E. nutans, Lolium perenne, and Leymus chinensis the seed coat prevented penetration of TTC. In contrast to these three species, a high proportion of intact $A$. sativa seeds (79\%) showed strong staining, suggesting that the seed coat in this species was permeable to TTC; piercing the seed coat resulted in a further increase in the proportion of staining (to 89\%) (Table 1).

\section{Relationships between EC, GP, and Field Emergence}

The relationship between EC and GP was analyzed in both intact and pierced seeds following controlled deterioration treatment. In intact seeds of the three species that possessed a semipermeable layer (E. nutans, Lolium perenne, and Leymus chinensis), there was no correlation $(P>0.05)$ between EC and GP $\left(R^{2}=0.0608\right.$, 0.0700 , and 0.0828, respectively) (Figures 1A-C). In pierced seeds of these species, however, EC showed a significant negative correlation $(P<0.01)$ with GP $\left(R^{2}=0.7708,0.8414\right.$, and 0.859 , respectively). In $A$. sativa, on the other hand, both intact and pierced seeds showed a significant negative correlation between EC and GP $\left(P<0.01 ; R^{2}=0.9071\right.$ and 0.9597 , respectively) (Figure 1D).

These results indicated that EC reflects seed germinability under laboratory conditions in A. sativa, but not in the other three species. This finding was borne out by a field experiment which showed that the EC value for intact A. sativa seed lots

TABLE 1 | Effect of piercing the seed coat on seed imbibition rate and on staining with triphenyl tetrazolium chloride (TTC), for four grass species.

\begin{tabular}{llcccc}
\hline \multirow{2}{*}{ Species } & & & \multicolumn{3}{c}{ Staining with TTC (\%) } \\
\cline { 4 - 6 } & Treatment & $\begin{array}{c}\text { Imbibition } \\
\text { (\%) }\end{array}$ & $\begin{array}{c}\text { No } \\
\text { staining }\end{array}$ & $\begin{array}{c}\text { Slight } \\
\text { staining }\end{array}$ & $\begin{array}{c}\text { Strong } \\
\text { staining }\end{array}$ \\
\hline Elymus nutans & Intact seeds & 67 & 83 & 17 & 0 \\
& Pierced seeds & 77 & 5 & 1 & 94 \\
Lolium perenne & Intact seeds & 65 & 100 & 0 & 0 \\
Leymus chinensis & Pierced seeds & 70 & 4 & 12 & 84 \\
& Intact seeds & 62 & 91 & 9 & 0 \\
Avena sativa & Pierced seeds & 89 & 9 & 2 & 89 \\
& Intact seeds & 40 & 5 & 16 & 79 \\
& Pierced seeds & 56 & 6 & 4 & 89
\end{tabular}

was negatively correlated with field emergence at the two sowing dates $\left[R^{2}=0.6069(P<0.01)\right.$ and $0.5316(P<0.05)$, respectively] (Figure 2B). However, there was no correlation between EC and field emergence in E. nutans at either date $\left(R^{2}=0.0025,0.0011\right.$; $P>0.05$, respectively) (Figure 2A).

\section{Optical Microscopy of Seed Coat and Endosperm}

The anatomy of the seed coat and endosperm for E. nutans, Lolium perenne, Leymus chinensis, and A. sativa is shown in Figure 3. In all four species, the structure of the seed coat adjacent to the endosperm was consistent with the basic characteristics of grass caryopsis, i.e., from the outside to the inside-pericarp, seed coat, aleurone layer, and starch. Moreover, the aleurone layer was in each case distinct and was stained positively with toluidine blue; however, the pattern of staining differed among species (Figures 3A-D). Thus, in E. nutans seeds, a black band was present between the pericarp and the aleurone layer (Figure 3A) and in Lolium perenne and Leymus chinensis seeds a white band was observable (Figures 3B,C); however, no band was observed in A. sativa (Figure 3D). It was deduced that this band represented the semipermeable layer and hence it was examined further by TEM and EDX.

\section{Determination of the Presence and Location of the Semipermeable Layer by TEM and EDX}

It was presumed that the semipermeable layer was the barrier that prevented lanthanum from penetrating further into the seed. As shown in Figure 4 (in which the black arrow indicates the location of lanthanum accumulation, whereas the white arrow indicates absence of lanthanum), clear lanthanum deposits were found in the pericarp and the testa (Figures $4 \mathbf{A}, \mathbf{C}, \mathbf{E}, \mathbf{G}$ ). Analysis of the internal parts of the seeds of E. nutans, Leymus chinensis, and Lolium perenne showed no lanthanum (Figures 4B,D,F), suggesting that the semipermeable layer (the band described above) prevented lanthanum penetration. However, lanthanum was observed in the aleurone layer of A. sativa seeds (Figure $\mathbf{4 H}$ ), suggesting that in this species no specific structure prevented lanthanum penetration. EDX analysis of the band showed that, whereas lanthanum was present in relatively large quantities in the seed coats of all the species (Figures $\mathbf{5 A}, \mathbf{C}, \mathbf{E}, \mathbf{G}$ ), there was no lanthanum in the endosperm of seeds of E. nutans, Lolium perenne, and Leymus chinensis (Figures 5B,D,F); however, it was present in the endosperm of $A$. sativa seeds (Figure $5 \mathbf{H}$ ). This indicated that lanthanum distribution was unrestricted in $A$. sativa seeds, consistent with the absence of a semipermeable layer in this species.

\section{DISCUSSION}

Seed vigor declines during seed storage on account of a series of changes, such as the accumulation of reactive oxygen species, lipid peroxidation, loss of cellular membrane integrity, enzyme 

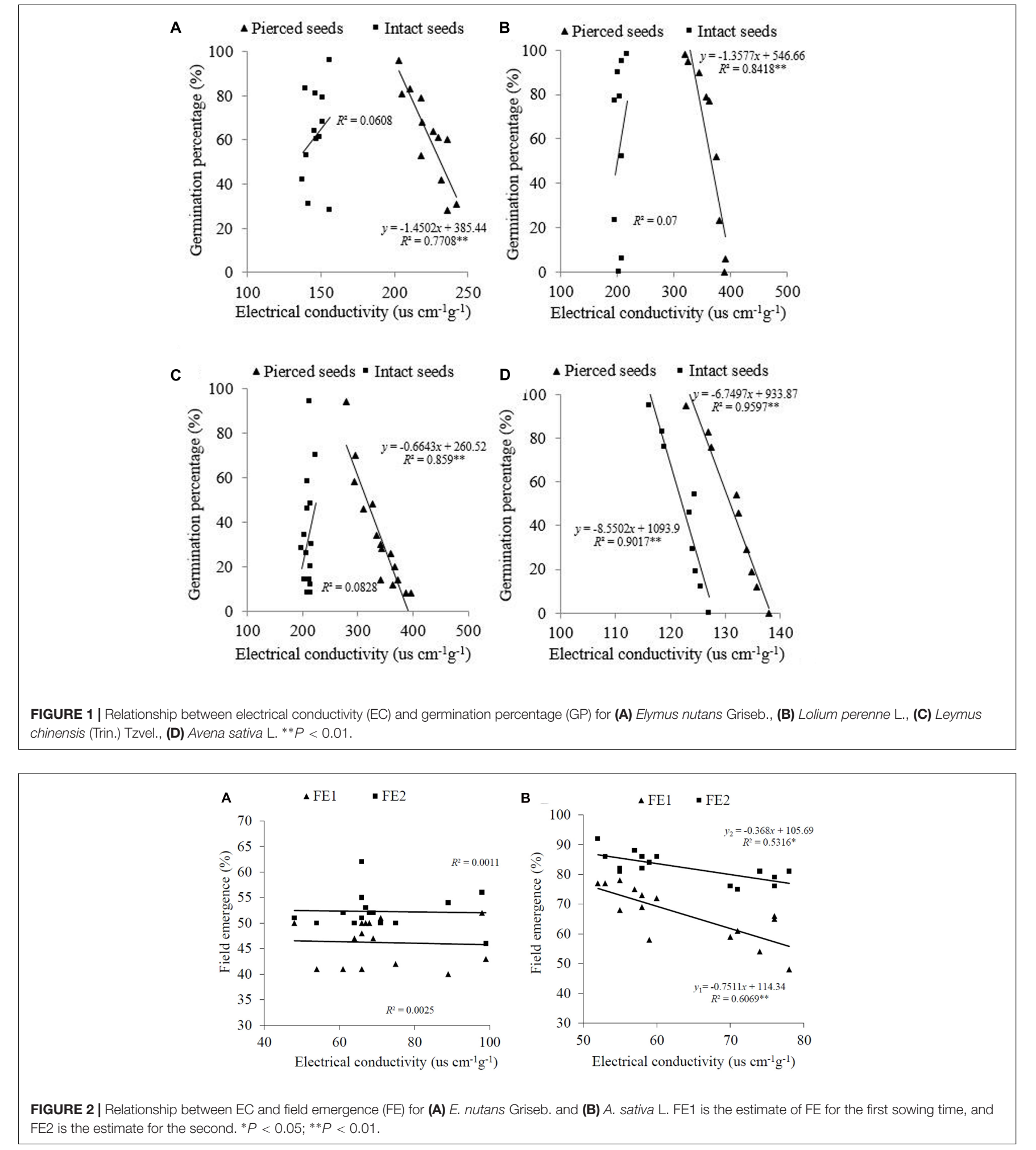

inactivation, weak energy metabolism, and DNA degradation (Kibinza et al., 2006; Parkhey et al., 2012; Ventura et al., 2012; Xin et al., 2014; Yin et al., 2014; Kong et al., 2015; Ratajczak et al., 2015; Wu et al., 2017). Generally, as seeds age, membrane integrity decreases and exosmosis occurs, and the EC value increases, thus reflecting seed vigor (Matthews and Bradnock, 1967; Hampton and TeKrony, 1995; Wang et al., 2002; Wang, 2003). 

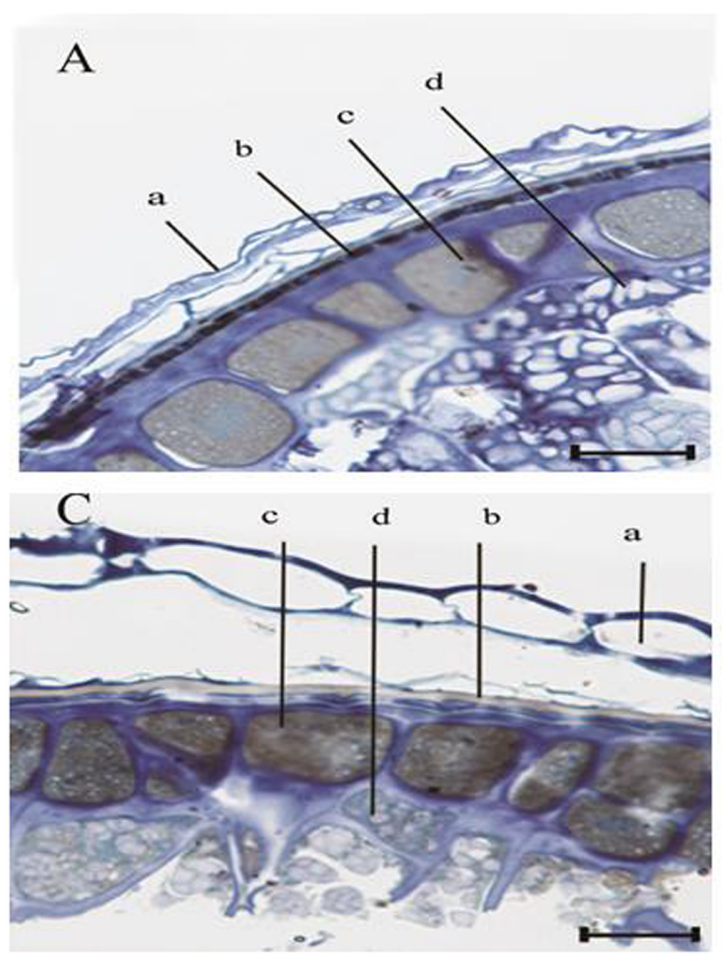
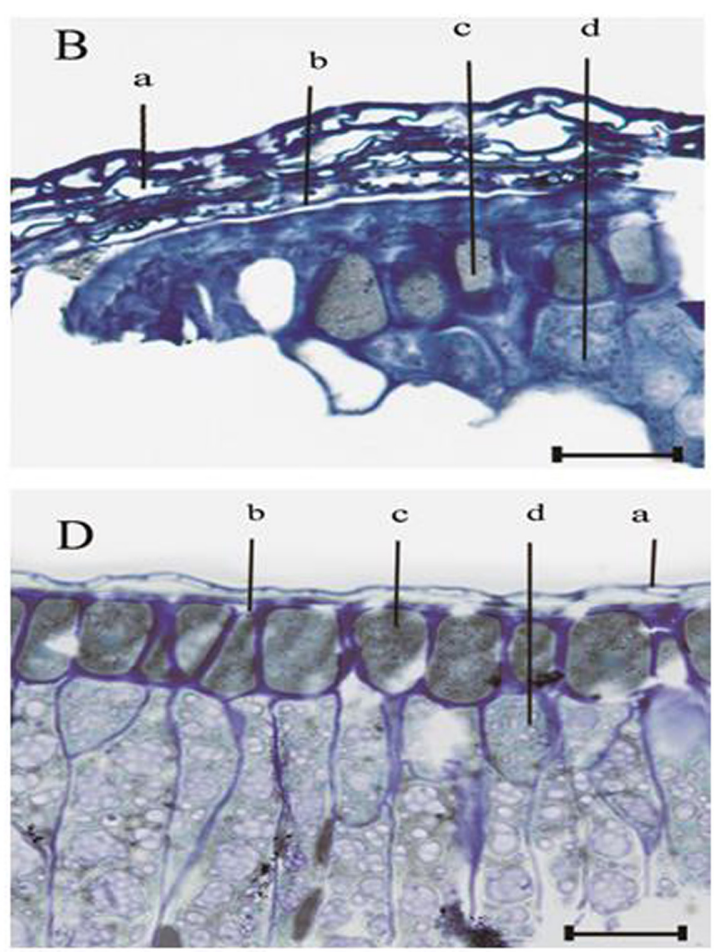

FIGURE 3 | Light micrograph of the seed coat adjacent to the endosperm, for four grasses. (A) E. nutans Griseb., (B) Lolium perenne L., (C) Leymus chinensis (Trin.) Tzvel., (D) A. sativa L. Scale bar = $25 \mu \mathrm{m}$; 'a' denotes the pericarp; 'b' denotes the testa; 'c' denotes the aleurone layer; and 'd' denotes the endosperm.
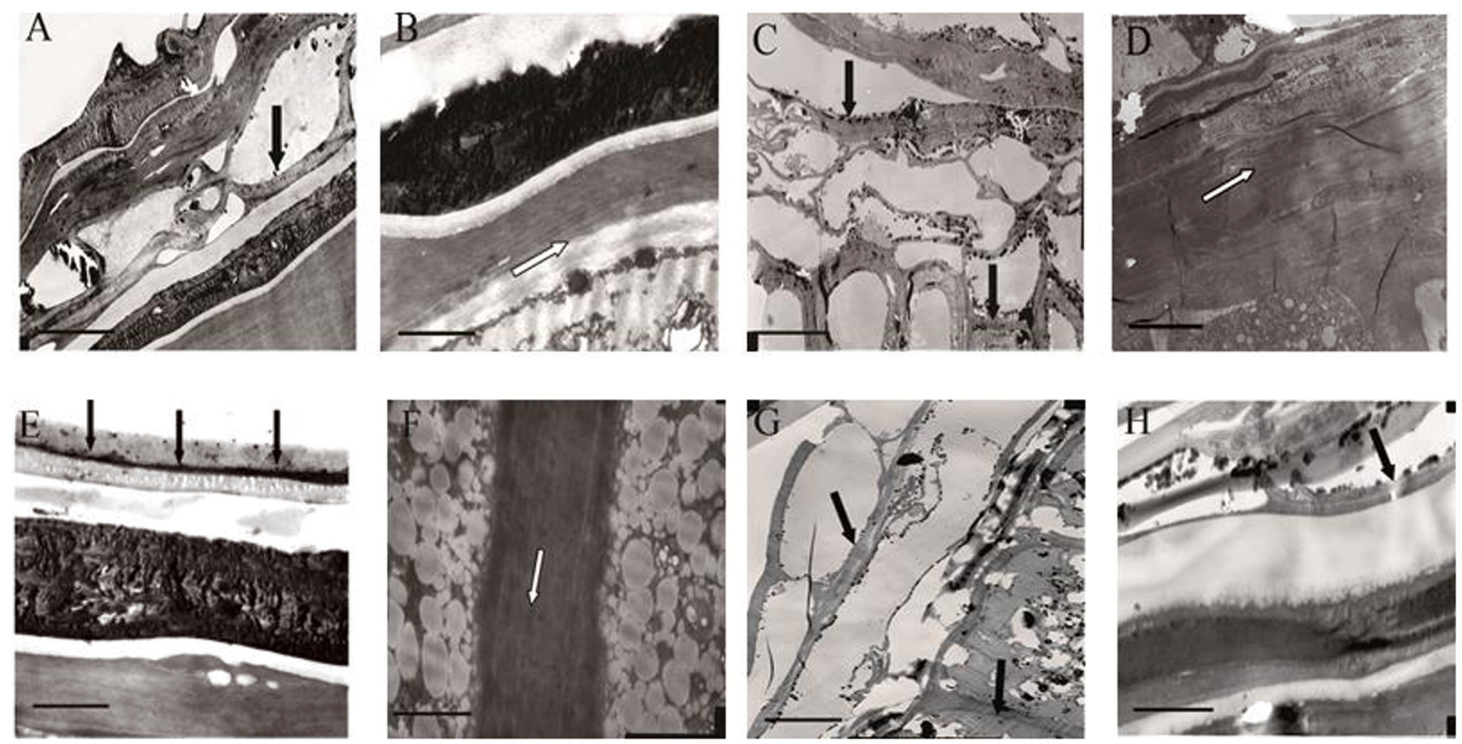

FIGURE 4 | Transmission electron micrograph (TEM) of the seed coat and endosperm cell membrane, for four grasses. E. nutans Griseb.: (A) Pericarp and testa, (B) Testa and aleurone layer; Lolium perenne L.: (C) Pericarp, (D) Seed coat and aleurone layer; Leymus chinensis (Trin.) Tzvel.: (E) Pericarp and testa, (F) Aleurone layer cell membrane; A. sativa L.: (G) Pericarp testa and aleurone layer, (H) Testa and aleurone layer. The scale bar for $\mathbf{A}, \mathbf{C}, \mathbf{E}, \mathbf{G}$ is $10 \mu \mathrm{m}$, and for $\mathbf{B , D}, \mathbf{F}, \mathbf{H}$ is $2 \mu \mathrm{m}$. Black dots are lanthanum granules, as indicated by black arrows.

The present study clearly showed that intact seeds of E. nutans, Lolium perenne, and Leymus chinensis exhibited little staining with TTC; and that in these species, no change in the EC value was observed during seed deterioration, although GP declined (Figures 1A-C). In contrast, in A. sativa, a high percentage of seeds showed TTC staining, and in intact seeds 

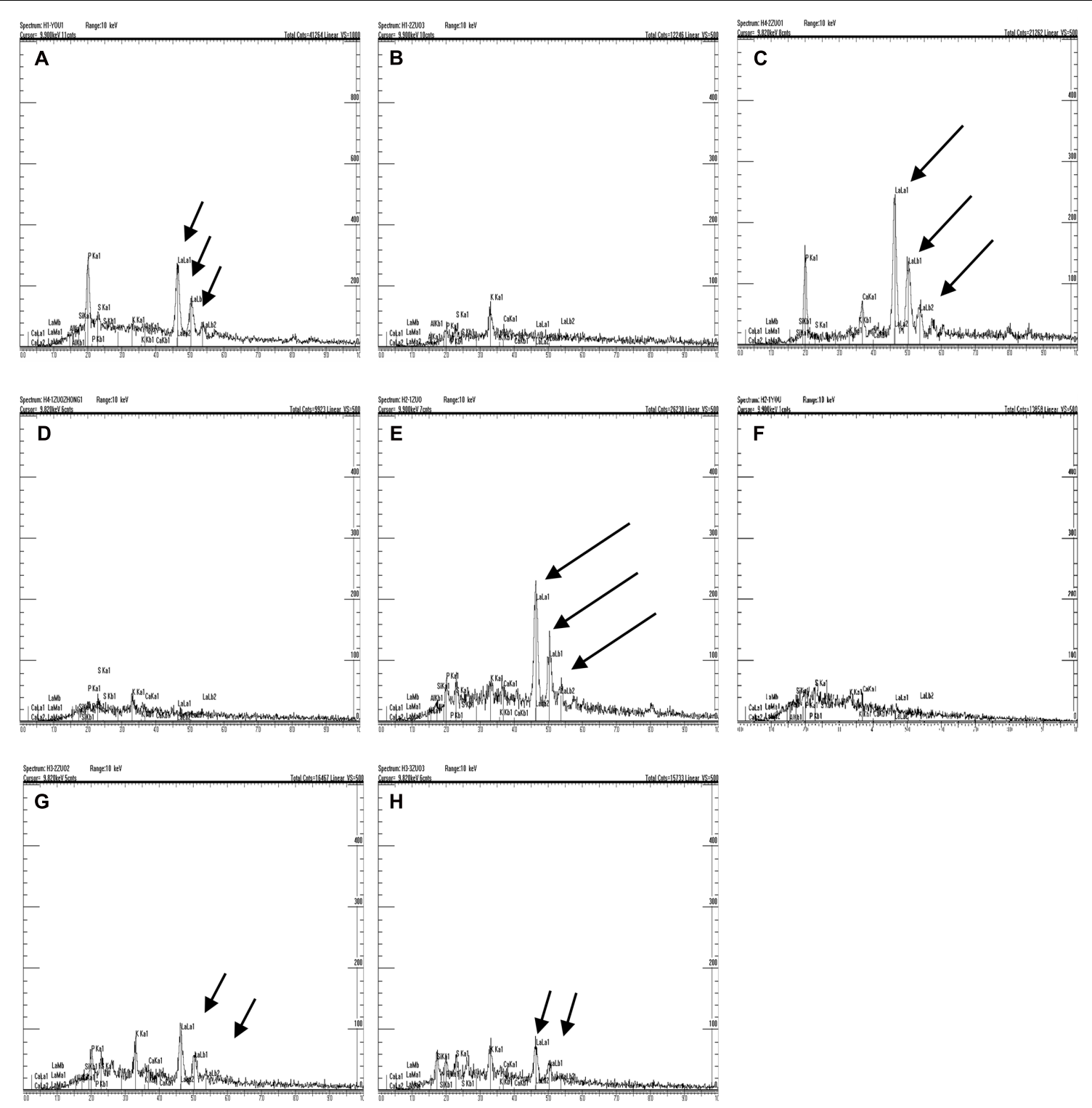

FIGURE 5 | Analysis of the semipermeable layer using energy-dispersive X-ray spectroscopy (EDX). Black arrows indicate the lanthanum peak. E. nutans Griseb.: (A) Testa, (B) endosperm; Lolium perenne L.: (C) Testa, (D) endosperm; Leymus chinensis (Trin.) Tzvel.: (E) Testa, (F) Endosperm; A. sativa L.: (G) Testa, (H) Endosperm.

of this species EC values were significantly negatively correlated with GP (Figure 1D). This suggests that in E. nutans, Lolium perenne, and Leymus chinensis, but not in A. sativa, there is a strong barrier that prevents solute leakage. Moreover, optical microscopy clearly suggested the presence of a semipermeable layer in the seed coats of E. nutans, Lolium perenne, and Leymus chinensis (Figures 3A-C), but not in that of A. sativa (Figure 3D). This was further supported by TEM and EDX analyses of lanthanum distribution following lanthanum nitrate infiltration, which showed that the distribution in the first three species did not extend beyond the seed (Figures 4B,D,E, 5B,D,F), whereas in A. sativa lanthanum was distributed internally, in the endosperm (Figures 4F, 5F).
Interestingly, for all species, piercing the seed coat resulted in a significant negative correlation between EC and GP (Figures 1A-D); i.e., as the EC value increased, GP declined. As compared to the behavior of intact seeds, cracks in the seed coat enhance both solute uptake and solute leakage. There are several factors affect EC value during seed deterioration (Yi et al., 1994; Han and Mao, 2000; Wang, 2003), among which, the semipermeable layer plays an important role, particularly. Piercing the seed coat loss integrity of the semipermeable layer and hence of the capacity to restrict solute movement.

Overall, it is apparent that, by reducing solute movement, the presence of a semipermeable layer negates the value of EC measurements in testing for seed vigor, in line with 
previous reports for seeds of Allium tuberosum, Allium cepa, Lycopersicon esculentum, and Capsicum annuum (Beresniewicz et al., 1995b; Taylor et al., 1995), and Sorghum sudanense (Wang, 2003; Wang et al., 2004). When a semipermeable layer is absent, for example in A. sativa, the EC value for intact seeds reflects seed germinability and seedling performance (see Figures 1D, 2B); the same observation has been made for Glycine max (L.) Merr. (Matthews and Bradnock, 1967; Loeffler et al., 1988), Brassica oleracea (Beresniewicz et al., 1995b; Taylor et al., 1995; Matthews et al., 2009), and F. arundinacea (Han et al., 1995). The phenomena of semipermeability appear to be widespread in seeds of higher plants. Previous researchers suggested that a seed semipermeable layer was present in 500 species belonging to 40 families; however, it was not found in the Leguminosae and in certain genera of the Cistaceae and Cruciferae (cited by Kotowski, 1927). The present study on grass species has shown for the first time that $A$. sativa does not possess a semipermeable layer. Similar results have been found for F. arundinacea (data not published). Although the location of the semipermeable layer varies with species (Beresniewicz et al., 1995a; Yan and Wang, 2008; Zhou et al., 2013a), its function is the same, which is to protect the mature embryo from drought stress (Prutsch et al., 2000), and to prevent or restrict the exchange of solutes whilst specifically permitting water and gas exchange to occur (Beresniewicz et al., 1995a). For many grasses, a semipermeable layer consisting of a cutin or suberin membrane in the caryopsis integuments restricts solute transport through the seed coat (Shull, 1913; Simpson, 1990). The degree of impermeability of the semipermeable layer may be related to its chemical composition (Beresniewicz et al., 1995b). Although seed aging causes some damage to membranes, it does not affect the semipermeable layer (Beresniewicz et al., 1995a,b; Yan, 2008). Taken together, these observations are all consistent with the function of the semipermeable layer as a strong barrier to restrict solute movement.

The significant negative correlation between EC and FE observed for A. sativa revealed that EC measurement was a useful predictor of seedling emergence in this species and provided a convenient and rapid method for seed vigor assessment. In contrast, the EC value was poorly correlated with field emergence in E. nutans (Figure 5A), which is consistent with results for Sorghum sudanense, Elymus sibiricus L. (Wang et al., 2004), Lolium perenne (Ching and Schoolcraft, 1968; Bennett et al., 1998), Lolium multiflorum Lam. (Marshall and Naylor, 1985), and Bromus biebersteinii (Hall and Wiesner, 1990). However,

\section{REFERENCES}

Bennett, J. S., Rowarth, J. S., and Jin, Q. F. (1998). Seed nitrogen and potassium nitrate influence browntop (Agrostis capillaris L.) and perennial ryegrass (Lolium perenne L.) vigor. J. Appl. Seed Prod. 16, 77-81.

Beresniewicz, M. M., Taylor, A. G., Goffinet, M. C., and Koeller, W. (1995a). Chemical nature of a semipermeable layer in seed coats of leek, onion (Liliaceae), tomato and pepper (Solanaceae). Seed Sci. Technol. 23, 135-145.

Beresniewicz, M. M., Taylor, A. G., Goffinet, M. C., and Terhune, B. T. (1995b). Characterization and location of a semipermeable layer in seed coats of leek after the seed coat had been pierced, the EC value clearly reflected seed germinability in E. nutans, Lolium perenne, and Leymus chinensis also: the higher the $\mathrm{EC}$ value, the lower the seed germinability (Figures $\mathbf{4 A - C}$ ). However, most grass species possess a seed semipermeable layer, and therefore TTC and EC values fail to estimate seed quality. The present study demonstrates that piercing the seed coat increases seed coat permeability, and suggests that EC measurements may then reflect seed germinability. However, whether EC values determined following piercing of the seed coat can predict seedling emergence for grass seeds possessing a semipermeable layer will require additional research.

\section{CONCLUSION}

A semipermeable layer is present in the seed coats of E. nutans, Lolium perenne, and Leymus chinensis. The inability of TTC staining or EC tests to assess seed quality in these three species is confirmed to be attributable to the presence of a semipermeable layer. In A. sativa, on the other hand, which lacks a semipermeable layer, TTC staining and EC measurement can be used as predictors of seed viability and vigor.

\section{AUTHOR CONTRIBUTIONS}

YL did part of the experiment and write the paper. XQH did another part of the experiment. YW provided the guidelines and reversed the manuscript several times and provided the funding. XWH made some comments in writing paper.

\section{FUNDING}

This work was supported by the National Basic Research Program (973 program) of China [grant number 2014CB138704], and the National Natural Science Foundation of China [grant number 30771532, 31672473].

\section{ACKNOWLEDGMENT}

We are very grateful to A.A. Powell for assistance in the preparation and revision of the manuscript.

and onion (Liliaceae), tomato and pepper (Solanaceae). Seed Sci. Technol. 23, $123-134$.

Ching, T. M., and Schoolcraft, I. (1968). Physiological and chemical differences in aged seeds. Crop Sci. 8, 407-409. doi: 10.2135/cropsci1968. 0011183X000800040003x

Finch-Savage, W. E. (1995). "Influence of seed quality on crop establishment, growth and yield," in Seed Quality: Basic Mechanisms and Agricultural Implications, ed. A. S. Basra (New York, NY: Food Product Press), 361-384.

Hall, R. D., and Wiesner, L. E. (1990). Relationship between seed vigor tests and field performance of 'Regar' meadow bromegrass. Crop Sci. 30, 967-970. doi: $10.2135 /$ cropsci1990.0011183X003000050001x 
Hampton, J. G., and TeKrony, D. M. (1995). Handbook of Vigor Tests Methods, 3rd Edn. Zurich: ISTA.

Han, J. G., and Mao, P. S. (2000). Changes of physiology and biochemistry during seed development of Siberian wildrye. Acta Agrestia Sin. 8, 237-244.

Han, J. G., Mao, P. S., Pu, X. C., and Li, M. (1995). A study on the seed vigor of tall fescue. Acta Agrestia Sin. 3, 269-275.

Hill, H. J., and Taylor, A. G. (1989). Relationship between viability, endosperm integrity and imbibed lettuce seed density and leakage. Hortscience 24, 814-816.

ISTA (2016). International Rules for Seed Testing. Bassersdorf: The International Seed Testing Association. doi: 10.15258/istarules.2016.F

Kibinza, S., Vinel, D., Côme, D., Bailly, C., and Corbineau, F. (2006). Sunflower seed deterioration as related to moisture content during ageing, energy metabolism and active oxygen species scavenging. Physiol. Plant. 128, 496-506. doi: $10.1111 / j .1399-3054.2006 .00771 . x$

Kong, L., Huo, H., and Mao, P. (2015). Antioxidant response and related gene expression in aged oat seed. Front. Plant Sci. 6:158. doi: 10.3389/fpls.2015.00158

Kotowski, F. (1927). Semipermeability of seed coverings and stimulation of seeds. Plant Physiol. 2, 177-186. doi: 10.1104/pp.2.2.177

Loeffler, T. M., TeKrony, D. M., and Egli, D. B. (1988). The bulk conductivity test as an indicator of soybean seed quality. J. Seed Technol. 12, 781-794.

Marshall, A. H., and Naylor, R. E. L. (1985). Seed vigor and field establishment in Italian ryegrass. Seed Sci. Technol. 13, 781-794.

Matthews, S., and Bradnock, W. T. (1967). Relationship between seed exudation and field emergence in peas and French beans. Hortic. Res. 8, 89-93.

Matthews, S., Demir, I., Celikkol, T., Kenanoglu, B. B., and Mavi, K. (2009). Vigour tests for cabbage seeds using electrical conductivity and controlled deterioration to estimate relative emergence in transplant modules. Seed Sci. Technol. 37, 736-746. doi: 10.15258/sst.2009.37.3.20

Parkhey, S., Naithani, S. C., and Keshavkant, S. (2012). ROS production and lipid catabolism in desiccating Shorea robusta seeds during aging. Plant Physiol. Biochem. 57, 261-267. doi: 10.1016/j.plaphy.2012.06.008

Prutsch, J., Schardt, A., and Schill, R. (2000). Adaptations of an orchid seed to water uptake and-storage. Plant Syst. Evol. 220, 69-75. doi: 10.1007/BF00985371

Ratajczak, E., Małecka, A., Bagniewska-Zadworna, A., and Kalemba, E. M. (2015). The production, localization and spreading of reactive oxygen species contributes to the low vitality of long-term stored common beech (Fagus sylvatica L.) seeds. J. Plant Physiol. 174, 147-156. doi: 10.1016/j.jplph.2014.08.021

Salanenka, Y. A., Goffinet, M. C., and Taylor, A. G. (2009). Structure and histochemistry of the micropylar and chalazal regions of the perispermendosperm envelope of cucumber seeds associated with solute permeability and germination. J. Am. Soc. Hortic. Sci. 134, 479-487.

Salanenka, Y. A., and Taylor, A. G. (2008). Seed coat permeability and uptake of applied systemic compounds. Acta Hortic. 782, 151-154. doi: 10.17660/ ActaHortic.2008.782.16

Shull, C. A. (1913). Semipermeability of seed coats. Bot. Gaz. 56, 169-199. doi: $10.1086 / 331142$

Simpson, G. M. (1990). Seed Dormancy in Grasses. New York, NY: Cambridge University Press, 114-119. doi: 10.1017/cbo9780511721816

Taylor, A. G., Lee, S. S., Beresniewicz, M. M., and Paine, D. H. (1995). Amino acid leakage from aged vegetable seeds. Seed Sci. Technol. 23, 113-122.
Ventura, L., Donà, M., Macovei, A., Crbonera, D., Buttafava, A., Mondoni, A., et al (2012). Understanding the molecular pathways associated with seed vigor. Plant Physiol. Biochem. 60, 196-206. doi: 10.1016/j.plaphy.2012.07.031

Wang, Y., Yu, L., Nan, Z. B., and Liu, Y. (2004). Vigor tests used to rank seed lot quality and predict field emergence in four forage species. Crop Sci. 44, 535-541. doi: $10.2135 /$ cropsci2004.5350

Wang, Y. R. (2003). Membrane Damage and During Seed Deterioration and Its Measurement and Control in Forage Species. Ph.D. thesis, Nanjing Agricultural University, Nanjing.

Wang, Y. R., Yu, L., and Liu, Y. L. (2002). Relationship between seed viability and membrane permeability during seed deterioration in several forage species. Acta Pratac. Sin. 11, 85-91.

Welbaum, G. E., and Bradford, K. J. (1990). Water relations of seed development and germination in muskmelon (Cucumis melo L.). IV. Characteristics of the perisperm during seed development. Plant Physiol. 92, 1038-1045. doi: 10.1104/ pp.92.4.1038

Wu, X., Ning, F., Hu, X., and Wang, W. (2017). Genetic modification for improving seed vigor is transitioning from model plants to crop plants. Front. Plant Sci. 8:8. doi: $10.3389 /$ fpls.2017.00008

Xin, X., Tian, Q., Yin, G., Chen, X., Zhang, J., Ng, S., et al. (2014). Reduced mitochondrial and ascorbate-glutathione activity after artificial ageing in soybean seed. J. Plant Physiol. 171, 140-147. doi: 10.1016/j.jplph.2013.09

Yan, X. J. (2008). Study of Semipermeable Layer on Several Grass Species. Masters thesis, Lanzhou University, Lanzhou.

Yan, X. J., and Wang, Y. R. (2008). Location of semipermeable layer and permeability of seed in Sorghum sudanense. Acta Pratac. Sin. 17, 54-59.

Yi, J., Cao, Z. C., Li, Q. F., and Wu, R. (1994). Problems and methods of testing seed vigor in Leguminosae and Gramineae. Grass Land China 3, 42-44.

Yin, G., Xin, X., Song, C., Chen, X., Zhang, J., Wu, S., et al. (2014). Activity levels and expression of antioxidant enzymes in the ascorbate-glutathione cycle in artificially aged rice seed. Plant Physiol. Biochem. 80, 1-9. doi: 10.1016/j.plaphy. 2014.03.006

Zheng, G. C., and Gu, Z. P. (1993). Book of Biological Microscopy. Beijing: People’s Education Press.

Zhou, J., Wang, Y. R., and Jahufer, Z. (2013a). Location and chemical composition of semi-permeable layer of forage seeds. Bangladesh J. Bot. 42, 23-29. doi: 10.3329/bjb.v42i1.15802

Zhou, J., Wang, Y. R., and Trethewey, J. (2013b). Semi-permeable layer formation during seed development in Elymus nutans and Elymus sibiricus. Acta Soc. Bot. Pol. 82, 165-173. doi: 10.5586/asbp.2013.012

Conflict of Interest Statement: The authors declare that the research was conducted in the absence of any commercial or financial relationships that could be construed as a potential conflict of interest.

Copyright (c) $2017 \mathrm{Lv}, \mathrm{He}, \mathrm{Hu}$ and Wang. This is an open-access article distributed under the terms of the Creative Commons Attribution License (CC BY). The use, distribution or reproduction in other forums is permitted, provided the original author(s) or licensor are credited and that the original publication in this journal is cited, in accordance with accepted academic practice. No use, distribution or reproduction is permitted which does not comply with these terms. 